\section{Concentraciones plasmáticas de linfopoyetina estromal tímica en niños infectados por virus dengue}

\section{Plasmatic concentrations of thymic stromal lymphopoietin in dengue virus-infected children}

\author{
David Pastrana ${ }^{1}$, Jhonny K. Muñoz ${ }^{1}$, \\ Felipe Ortiz ${ }^{1}$, Rocío Vega ${ }^{2}$, \\ Jairo A. Rodríguez ${ }^{2}$, Doris Salgado ${ }^{2}$, \\ Luz Stella Rodríguez ${ }^{3}$ y Carlos F. Narváez ${ }^{2}$
}

Palabras clave: virus dengue (VD),

linfopoyetina

estromal tímica

(TSLP),

linfocitos T (LT),

Th2, ELISA

Key words:

virus dengue (VD),

thymic stromal

lymphopoietin,

(TSLP),

T lymphocytes, Th2 ELISA

\section{Resumen}

La linfopoyetina estromal tímica (TSLP) es un citoquina recientemente descrita que juega un papel clave en la fisiopatología de enfermedades alérgicas y parasitarias. La TSLP, luego de ser producida por el epitelio en respuesta a diferentes estímulos como componentes bacterianos y virales, condiciona a células dendríticas locales a generar una respuesta de linfocitos Th2. En el dengue, una enfermedad viral febril endémica en Colombia, un predominante perfil de citoquinas Th2 ha sido descrito en los niños que presentan formas severas y potencialmente letales de la enfermedad. Aquí se determinaron por ELISA, las concentraciones plasmáticas de TSLP en niños con dengue en fase aguda y convaleciente de la enfermedad, comparándolos con los niveles presentes en niños sanos y atópicos como controles. Los resultados indican que no hay diferencias significativas en las concentraciones plasmáticas de TSLP entre los niños sanos y los niños infectados con dengue en fase aguda. Sin embargo, una disminución significativa fue encontrada en los niños infectados entre la etapa aguda y convaleciente de la infección. Cuando se compararon los niveles de TSLP con el número de plaquetas, un conocido marcador de severidad en el dengue, no hubo correlación. Estos hallazgos apoyan la hipótesis de que el TSLP plasmático podría no estar implicado directamente en la fisiopatología de la infección por virus dengue. Otros factores de polarización de linfocitos T locales y sistémicos deberían ser evaluados.
Abstract
Thymic stromal lymphopoietin (TSLP) is a recently described cytokine involved in the physiopathogenesis of allergic and parasitic diseases. After production by epithelial cells in response to different stimuli like bacterial and viral components, the TSLP modulate the function of dendritic cells to generate a Th2-lymphocyte response. In dengue, an endemic viral febrile disease in Colombia, a predominant Th2-cytokine pattern has been described in children suffering forms severe and potentially lethal dengue. Here, it was determined by ELISA, the plas- ma concentrations of TSLP in children undergoing the acute and convalescent stage, in comparison to healthy and atopic children's levels as controls groups. Results show no significant differences in plasma concentrations of TSLP between healthy and acute-phase dengue-infected

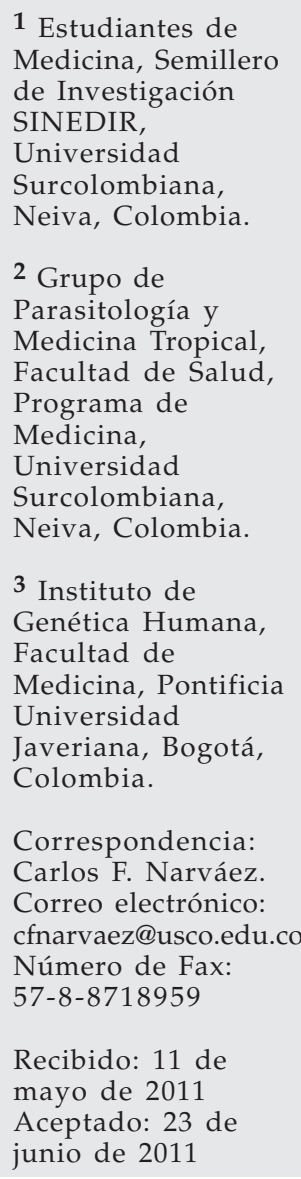

1 Estudiantes de Medicina, Semillero de Investigación SINEDIR, Universidad Surcolombiana, Neiva, Colombia.

2 Grupo de Parasitología y Medicina Tropical, Facultad de Salud, Programa de Medicina, Universidad Surcolombiana, Neiva, Colombia.

3 Instituto de Genética Humana, Facultad de Medicina, Pontificia Universidad Javeriana, Bogotá, Colombia.

Correspondencia: Carlos F. Narváez. Correo electrónico: cfnarvaez@usco.edu.co Número de Fax: 57-8-8718959

Recibido: 11 de mayo de 2011 Aceptado: 23 de junio de 2011 
children and just atopic children showed significant higher levels of TSLP. However, a significant decrease in plasmatic TSLP was noted between the acute and convalescent phase of the dengue infection. When comparing the TSLP levels to the number of platelets, a known marker of dengue severity, there was no correlation. These findings support the hypothesis that plasma TSLP could not be directly involved in the pathogenesis of dengue infection in children and other local Tlymphocyte polarization and systemic factors should be explored.

\section{Introducción}

El dengue es la enfermedad viral transmitida por vector más importante a nivel mundial. Se estima que 2,5 billones de personas en el mundo están en riesgo de contraer la infección y que más de 50 millones se infectan cada año, número que incluye a los 500.000 casos en donde la hospitalización es necesaria. En general, la mortalidad por dengue se estima en $1 \%$, pero varía dependiendo de la región analizada y puede llegar a ser del $5 \%$ en países como el nuestro $^{(1)}$. El virus dengue (VD) es un virus que pertenece a la familia Flaviviridae, cuyo genoma está constituido por ARN de cadena sencilla y polaridad positiva. Existen cuatro serotipos diferentes (DEN-1, DEN-2, DEN-3 y DEN-4) con una homología mayor al $65 \%$ entre ellos. El virión maduro está conformado por 3 proteínas estructurales: la proteína E que se encuentra en la envoltura, proteína $\mathrm{M}$, asociada a la membrana, proteína $C$ que forma la cápside y 7 proteínas no estructurales (NS1, NS2a, NS2b, NS3, NS4a, NS4b y NS5) que especialmente participan en el control de la replicación viral y en la evasión de la respuesta inmune (2-4). $^{(2)}$

La infección con VD causa una enfermedad de amplio espectro clínico que incluye desde cuadros asintomáticos hasta cuadros graves que pueden conducir a la muerte. Entre las formas graves se destacan hemorragias, choque, insuficiencia hepática, encefalopatía y miocarditis ${ }^{(5,6)}$. Actualmente se han dilucidado algunos de los mecanismos por los cuales la infección por VD puede provocar una enfermedad severa. Entre ellos se destacan la alteración de la inmunidad mediada por células con el aumento de producción de mediadores inflamatorios, la amplificación dependiente de anticuerpos (ADA) y la acción del complemento ${ }^{(7,8)}$.
Hay evidencia de que el patrón de citoquinas producido por los linfocitos T (LT) durante la infección, es un factor determinante de la severidad de la enfermedad(9). Se ha reportado por ejemplo, que LT CD8+ homotípicos producen generalmente importantes cantidades de IFN $\gamma$, un citoquina clave para la inmunidad celular, mientras que LT CD8 + heterotípicos generalmente producen importantes cantidades de $\mathrm{TNF} \alpha$, una citoquina proinflamatoria que ha sido asociada con la severidad de la enferme$\operatorname{dad}^{(10,11)}$. Mientras que el papel de las citoquinas en la fisiopatología y asociación con manifestaciones clínicas de la enfermedad permanece controversial, es aceptado que el IFN $\gamma$ puede tener un papel protector en la infección o vacunación con $\mathrm{VD}^{(12)}$. Por el contrario, se ha descrito que en niños que sufren formas severas de infección por VD ocurre un cambio hacia la producción de IL4 (clásica citoquina que define el perfil Th2) ${ }^{(13)}$.

Varios factores inducen la polarización de LT vírgenes hacia un perfil Th2. Dentro de ellos tenemos la baja producción de IL-12p70 por la célula presentadora de antígeno (CPA), la presencia de IL-4 externa, la relación del número de CPA y LT, entre otras ${ }^{(14)}$. Recientemente la TSLP ha sido mostrada como uno de los principales factores que inducen a la célula dendrítica (CD) a producir la polarización de LT hacia un perfil Th2. En apoyo de esto, la TSLP ha sido ampliamente caracterizada en enfermedades alérgicas y parasitarias ${ }^{(15-17)}$. Las células epiteliales de la piel, intestino y pulmones son la principal fuente de TSLP en el humano; adicionalmente otros tipos celulares como fibroblastos, mastocitos, células de musculo liso bronquial y recientemente células dendríticas murinas, pueden bajo 
ciertas condiciones de inflamación producir TSLP $(15,18-20)$. Algunos estímulos como citoquinas, componentes bacterianos como el ácido lipoteicoico, lipopolisacáridos (LPS), peptidoglicano y componentes estructurales virales (como el ARN de doble cadena) son inductores de la producción de TSLP por el epitelio(21-23). El efecto inductor en la producción de TSLP por reconocidos patrones moleculares asociados a patógenos es posiblemente mediado a través de los Toll-like receptors (TLR, por sus siglas en inglés) ${ }^{(24)}$. La CPA local, generalmente una célula dendrítica, es condicionada por el TSLP para generar una respuesta Th2 cuando migra al nódulo linfoide secundario(16,25). Este condicionamiento es especialmente caracterizado por una disminución en la producción de IL12 p70 y un aumento en la producción de IL-10 por parte de la célula dendrítica ${ }^{(26)}$. Finalmente, en todos estos modelos, la producción de TSLP favorece la respuesta Th2.

Ya que en formas severas de dengue ha sido mostrada la existencia de una respuesta Th2 y que uno de los más fuertes factores inmunes recientemente asociado a la polarización Th2 in vitro e in vivo es la TSLP, en este trabajo se propuso determinar si los niveles de TSLP en plasma están alterados durante la infección por VD en niños.

\section{Materiales y métodos}

\section{Pacientes y muestras}

Niños con edades entre 1 y 14 años, fueron divididos en tres grupos, cada uno con un total de 20 integrantes: 1) niños sanos, 2) niños atópicos, 3) niños con dengue. Como niños sanos fueron tomados los menores provenientes de una Institución Educativa, previo examen pediátrico y paraclínico (cuadro hemático). El grupo de pacientes atópicos se conformó a partir de los niños que asistían a la consulta de alergia del Hospital Universitario de Neiva y que tenían al menos uno de los siguientes diagnóstico clínico y/ o paraclínico: eczema, rinitis y/o asma. En el grupo de niños con dengue se incluyeron pacientes hospitalizados en el servicio de pediatría del Hospital Universitario de Neiva que cumplían con criterios diagnósticos según la clasificación revisada de la Organización Mundial de la Salud ${ }^{(27)}$. De los 20 niños incluidos en el grupo de dengue, 19 tenían diagnóstico de dengue con signos de alarma y 1 niño correspondió a dengue severo. Los signos de alarma (n) en el grupo de dengue fueron: dolor abdominal (11), vómito persistente (6), trombocitopenia marcada (2). La característica clínica del paciente con dengue severo fue el choque.

A todos los niños se les tomó 4 a $6 \mathrm{~mL}$ de sangre total, por venopunción, en tubos con EDTA. Posteriormente la muestra fue centrifugada a $250 \mathrm{xg}$ por 10 minutos, se tomó el plasma que fue conservado a $-20^{\circ} \mathrm{C}$ hasta el momento de la realización del ELISA para la cuantificación de TSLP. Al grupo de niños con dengue se les tomó una muestra en la fase aguda (día 3 a 7 después de iniciada la fiebre) y otra en la fase de convalecencia (día 14).

El protocolo utilizado en este trabajo fue aprobado por el Comité de Ética de la Facultad de Salud de la Universidad Surcolombiana. Todos los padres o acudientes firmaron el consentimiento informado.

\section{Diagnóstico de infección por VD}

El diagnóstico de infección por VD y la clasificación se realizó teniendo en cuenta los criterios revisados de la OMS ${ }^{(27)}$. El diagnóstico clínico de la infección se apoyó por la presencia de IgM específica para VD y/o la proteína viral NS1 ${ }^{(28)}$ en el plasma tomado después del día 6 o al día 3 día de iniciados los síntomas, respectivamente.

\section{Detección de TSLP en plasma}

La detección de la concentración plasmática de TSLP se realizó por medio de ELISA, usando un estuche comercial (R\&D systems, número de catálogo: DY1398), siguiendo las recomendaciones del fabricante. Este estuche ha sido utilizado en previas publicaciones para la medición de TSLP en modelos de infección viral ${ }^{(29)}$ y en niños con 
síndrome atópico ${ }^{(30)}$. Brevemente, placas de 96 pozos (Nunc, Thermo Scientific, USA) se cubrieron con anticuerpo anti-TSLP humano hecho en oveja y se incubaron a temperatura ambiente toda la noche. Al día siguiente, se desechó el sobrenadante y se realizaron 3 lavados con PBS - Tween 20 al 0,05\% (solución de lavado), se bloqueó con PBS - albúmina sérica bovina (BSA) al 1\% (reactivo diluyente) y se incubaron a temperatura ambiente por $1 \mathrm{~h}$. Tras lavar en tres oportunidades con solución de lavado, se adicionaron $100 \mu \mathrm{L} /$ pozo de las muestras en diluciones seriadas. Como control negativo se agregó a algunos pozos reactivo diluyente en ausencia de muestra. La placa fue cubierta e incubada a temperatura ambiente por $2 \mathrm{~h}$ al cabo de las que se repitió el lavado de las placas, se adiciono el anticuerpo de detección anti-TSLP humano biotinilado y se incubó por $2 \mathrm{~h}$ más. Después de tres lavados, se agregó estreptavidina-peroxidasa y se incubó por 20 minutos, se repitió el proceso de lavado y se agregó $100 \mu \mathrm{L} /$ pozo de solución de revelado (1 tableta de tetrametilbenzidina, $1 \mathrm{~mL}$ de DMSO, $9 \mathrm{~mL}$ de buffer citrato fosfato y $10 \mu \mathrm{L}$ de peróxido de hidrógeno). Las placas fueron detenidas con $50 \mu \mathrm{L}$ de $\mathrm{H}_{2} \mathrm{SO}_{4}, 2 \mathrm{~N}$. La placa fue leida usando un espectrofotómetro ELX-800 (BioTek instruments), a una longitud de onda de $450 \mathrm{~nm}$. El límite de sensibilidad del ensayo es de $31,2 \mathrm{pg} / \mathrm{mL}$.

\section{Análisis estadístico}

Todos los resultados son mostrados en forma de mediana (rango). Para el análisis estadístico se utilizó el software GraphPadPrism versión 5.0. Pruebas estadísticas no paramétricas fueron usadas. Para el análisis de dos grupos independientes se utilizó la prueba de MannWhitney y la prueba de Wilcoxon para el análisis de datos pareados. Para determinar el grado de correlación entre dos variables se usó el coeficiente de correlación de Spearman (rho). Una $\mathrm{P} \leq 0,05$ fue considerada estadísticamente significativa en todos los casos. Para los valores de TSLP por debajo del límite de sensibilidad y por propósitos estadísticos, se les asignó un valor de $15 \mathrm{pg} / \mathrm{mL}$.

\section{Resultados}

\section{Concentraciones plasmáticas de TSLP en los pacientes con dengue}

Debido a que un patrón de citoquinas Th2 ha sido asociado con formas severas de dengue $^{(13)}$ y la TSLP ha sido un factor que favorece la respuesta $\mathrm{Th} 2^{(16)}$, se determinaron los niveles plasmáticos de TSLP en niños infectados con dengue y se compararon con niños sanos y atópicos como control.

Como muestra la Figura 1, no existieron diferencias significativas en la concentración de TSLP entre niños sanos y los infectados con dengue en fase aguda, con una mediana (rango) de 50,9 pg/mL (15,6-942) y 50,5 pg/ $\mathrm{mL}(15,6-1,228,3)$, respectivamente $(P=0,76$, Mann-Whitney test). Como ha sido previamente reportado, se observó un aumento significativo de las concentraciones de TSLP en niños atópicos al ser comparados con niños sanos ( $P=0,032$, Mann-Whitney test) y niños con dengue ( $\mathrm{P}=0,047$, Mann-Whitney test).

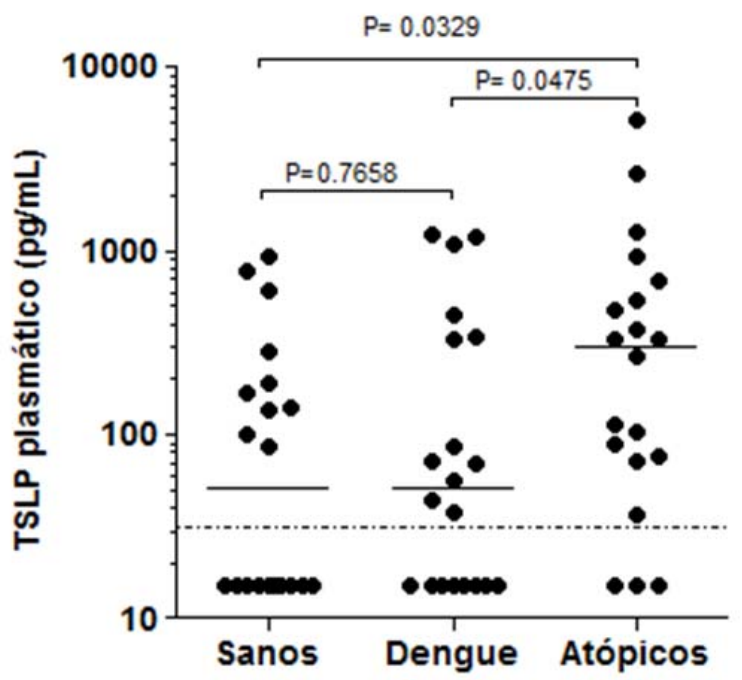

Figura 1. Concentración de TSLP en plasma. Los niveles de TSLP en las muestras de plasma de niños sanos, niños infectados con VD en fase aguda y niños atópicos fueron evaluadas por ELISA. Los puntos representan los niveles de TSLP de cada niño. Las barras horizontales representan la mediana. El valor de $\mathrm{P}$ usando la prueba de Mann-Whitney es mostrado. La línea punteada representa el nivel de sensibilidad del ensayo $(31,2 \mathrm{pg} / \mathrm{mL})$. 
Diecisiete de 20 niños atópicos, tuvieron concentraciones detectables de TSLP, con una mediana de $299 \mathrm{pg} / \mathrm{mL}(15,6-5,163)$. En conjunto estos resultados sugieren que la infección con VD en su fase aguda, no altera la concentración plasmática de TSLP y que niños con atopía tienen incrementado el nivel de este marcador.

Niveles de TSLP plasmático en fase aguda y convaleciente de la enfermedad por VD

Ya que los valores plasmáticos de las citoquinas son dinámicos y varían rápidamente a través del tiempo, la concentración de TSLP fue evaluada en fase aguda y convaleciente de la infección en los mismos pacientes infectados con dengue.

Como se muestra en la Figura 2, una caída significativa en la cantidad de TSLP circulante fue detectada entre la etapa aguda y convaleciente de la infección, con una mediana (rango) de 50,5 pg/mL $(15,6-1,228)$ y $15,6 \mathrm{pg} / \mathrm{mL}(15,6-1,177)(\mathrm{P}=0,021$, Wilcoxon test), respectivamente. En conjunto estos resultados sugieren que en la convalecencia de los pacientes que se recuperaron de una infección por VD, la cantidad TSLP fue inhibida.

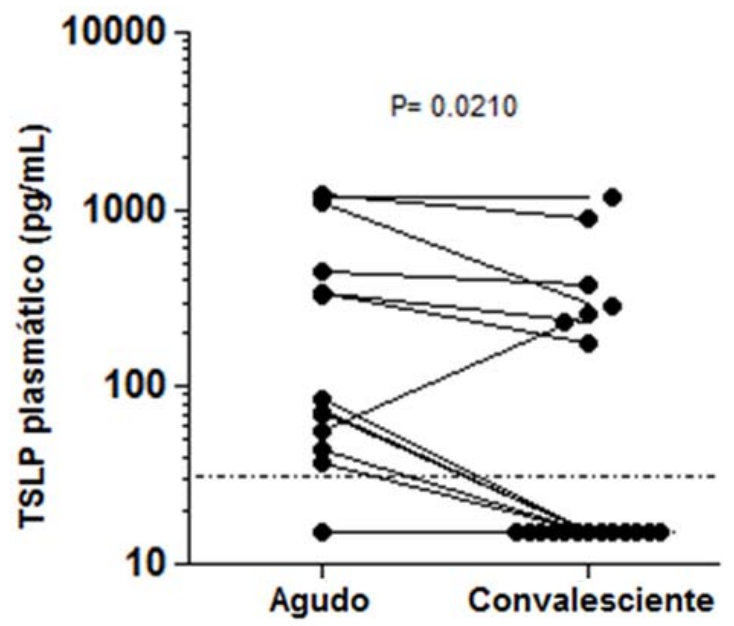

Figura 2. Concentración plasmática de TSLP en niños infectados por VD en fase aguda y convaleciente de la infección. Las concentraciones de TSLP en el plasma de los niños infectados con VD fueron evaluadas por ELISA. Las líneas continuas unen la muestra tomada en fase aguda y de convalecencia en el mismo paciente. La P establecida usando la prueba de Wilcoxon es mostrada. La línea punteada representa el nivel de sensibilidad del ensayo $(31,2 \mathrm{pg} / \mathrm{mL})$.
La concentración de TSLP plasmática no se asocia con el número de plaquetas circulantes

Finalmente se evaluó la posible asociación entre la concentración de TSLP y la trombocitopenia como marcador de gravedad de la enfermedad. El número de plaquetas se obtuvo del cuadro hemático de los pacientes realizado en la misma muestra en que la TSLP fue medida. Los resultados obtenidos (Figura 3) no mostraron correlación alguna entre la concentración plasmática de TSLP y el grado de trombocitopenia en los pacientes con dengue (Spearman rho = $0,18, \mathrm{P}=0,42)$. Este resultado sugiere que la TSLP no está relacionada con el descenso en las plaquetas observado en los pacientes infectados con VD.

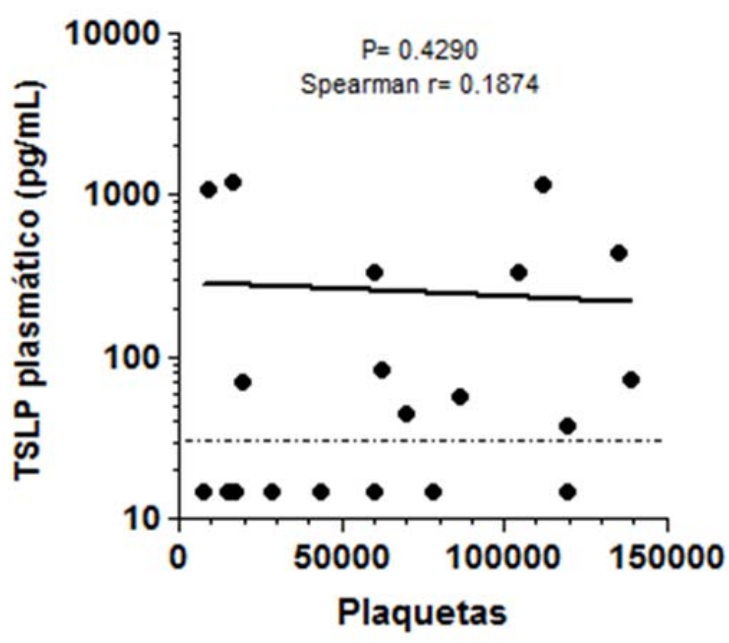

Figura 3. Ausencia de correlación entre los niveles de TSLP y el conteo de plaquetas circulantes. Los niveles de TSLP y número de plaquetas fueron evaluados en la misma muestra en fase aguda. La línea de tendencia y el grado de correlación (RHO de Spearman) son mostrados.

\section{Discusión}

En este estudio se analizó la concentración plasmática de TSLP en niños infectados con VD, niños sanos y niños atópicos como controles y se encontró que: 1 . No existieron diferencias en la concentración plasmática de TSLP entre niños infectados con VD y niños sanos, pero fue significativamente más alta en los niños atópicos. 
2. Hubo una caída significativa de la TSLP entre la fase aguda y de convalecencia de los niños infectados con VD. 3. No se encontró correlación entre los niveles de TSLP plasmático y el número de plaquetas circulantes.

Al comparar los niveles plasmáticos de TSLP entre niños infectados con VD, niños sanos (como control negativo) y niños atópicos (como control positivo), no se encontraron diferencia estadísticamente significativa entre los dos primero. Como se esperaba y en apoyo de datos previamente publicados ${ }^{(30)}$, niveles significativamente más altos de TSLP fueron encontrados en niños atópicos. Estos resultados apoyan la validez del ensayo usado para la detección de TSLP plasmático en este estudio, sugiriendo la no participación de éste factor circulante en la fisiopatología de la infección por VD. De notar, los niveles basales de TSLP encontrados en niños sanos fueron superiores a los reportados recientemente en una población pediátrica asiática ${ }^{(30)}$. Niveles diez veces más altos de TSLP fueron también encontrados en los niños atópicos de nuestro estudio comparado a niños atópicos asiáticos. Helmintiasis adquiridas en edad pediátrica, pueden modular la respuesta inmune a alérgenos y a la vacunación ${ }^{(31)}$. Es posible, como es frecuente en países en vías de desarrollo, que las infecciones parasitarias crónicas del tracto gastrointestinal y que escapan al examen médico rutinario, predispongan en la población pediátrica la liberación de cantidades significativas de TSLP al plasma de forma "basal", haciendo por lo tanto que la TSLP se detecte de manera más evidente en estos niños. Otros factores como los genéticos no deben ser descartados como explicación a las diferencias en los valores de TSLP plasmáticos encontrados en estas dos poblaciones ${ }^{(32)}$.

Existió una caída significativa en la concentración de TSLP entre la fase aguda y convaleciente de la infección por VD. La razón que explica esta diferencia no es clara. La gran mayoría de los niños infectados incluidos en este estudio tienen diagnóstico de dengue con signos de alarma y todos ellos por lo tanto, no desarrollaron dengue severo. El IFN $\gamma$ (marcador típico de la respuesta
Th1) ha sido implicado en la protección contra las formas severas de la infección e incluso en modelos humanos de vacunación, LT CD8+ productores de IFN han sido recientemente propuestos como un marcador de protección ${ }^{(33)}$. Es posible que la mayoría de estos niños que no desarrollaron la forma severa hubieran tenido un perfil Th1 dominante que haya por tanto suprimido durante el curso tardío de la enfermedad factores que inducen la respuesta $\mathrm{Th} 2$, dentro de ellos la TSLP.

En apoyo de la no participación directa del TSLP plasmático en la fisiopatología de la infección por VD, no se encontró correlación entre los niveles de TSLP y la trombocitopenia, un conocido marcador de severidad ${ }^{(27)}$, aunque es claro que este no es el estudio ideal para analizar el posible papel del TSLP en la severidad de la infección puesto que solo un paciente cumplió con los criterios de dengue severo. Sin embargo, los niveles de TSLP en el plasma de ese paciente se encontraron también por debajo del límite de detección de la técnica.

Aunque son muchas las publicaciones sobre TSLP y modelos experimentales que muestran su implicación en procesos inmunológicos, son relativamente escasos aquellos trabajos que miden el comportamiento de la TSLP en plasma humano y esto se limita a enfermedades atópicas ${ }^{(30)}$; Este es el primer trabajo, a nuestro conocimiento, que explora el papel de este mediador inmune medido en plasma en la fisiopatología de la infección por VD y los resultados en general sugieren que no hay una implicación directa de este.

Existen otras citoquinas que juegan también un importante papel en la inducción de un perfil Th2, como lo son la IL-7, IL-33 y la IL-25(34,35), que bajo estímulos de algunos componentes de patógenos pueden iniciar un cambio en la respuesta inmunológica y por tanto deben ser evaluados en la infección por VD en un futuro. La participación de la TSLP en la respuesta inmune a VD debería además ser analizada no solo a nivel sistémico (como el evaluado aquí) sino a nivel local en el epitelio. 


\section{Agradecimientos}

A los pacientes, a los niños sanos, a la Institución Educativa Angel María Paredes, al personal de pediatría del Hospital Universitario de Neiva y al semillero de investigación SINEDIR por su apoyo al estudio.

Este trabajo fue financiado por la Vicerrectoría de Investigación y Proyección Social de la Universidad Surcolombiana, mediante la convocatoria interna de proyectos de menor cuantía y apoyo a Semilleros de Investigación.

\section{Referencias Bibliográficas}

1. Guzmán, M.G. \& Kouri, G. 2009. Dengue in Cuba: research strategy to support dengue control. Lancet 374: 1660-1661, doi: S01406736(09)61975-9 [pii] 10.1016/S0140-6736 (09)61975-9.

2. Perera, R. \& Kuhn, R.J. 2008. Structural proteomics of dengue virus. Curr Opin Microbiol 11: 369-377, doi: S1369-5274(08) 00089-1 [pii] 10.1016/j.mib.2008. 06.004.

3. Ashour, J. et al. 2010. Mouse STAT2 restricts early dengue virus replication. Cell Host Microbe 8: 410-421, doi: S1931-3128(10) 00344-6 [pii] 10.1016/j.chom.2010.10.007.

4. Munoz-Jordan, J.L. et al. 2005. Inhibition of alpha/beta interferon signaling by the NS4B protein of flaviviruses. J Virol 79: 8004-8013, doi: 79/13/8004 [pii] 10.1128/JVI.79.13. 8004-8013.2005.

5. Salgado, D.M., Panqueba, C.A., Castro, Castro, D., M, R.V. \& Rodríguez, J.A. 2009. [Myocarditis in children affected by dengue hemorrhagic fever in a teaching hospital in Colombia]. Rev Salud Pública (Bogotá) 11: 591600, doi: S0124-00642009000400010 [pii].

6. Salgado, D.M. et al. Heart and skeletal muscle are targets of dengue virus infection. Pediatr Infect Dis J 29: 238-242, doi: 10.1097/INF. $0 \mathrm{~b} 013 \mathrm{e} 3181 \mathrm{bc} 3 \mathrm{c} 5 \mathrm{~b}$.

7. Nielsen, D.G. 2009. The relationship of interacting immunological components in dengue pathogenesis. Virol J 6: 211, doi: 1743422X-6-211 [pii] 10.1186/1743-422X-6-211.

8. Murphy, B.R. \& Whitehead, S S. 2011. Immune response to dengue virus and prospects for a vaccine. Annu Rev Immunol 29: 587-619, doi: 10.1146/annurev-immunol031210-101315.

9. Hatch, S. et al. 2011. Intracellular cytokine production by dengue virus-specific $\mathrm{T}$ cells correlates with subclinical secondary infection. J Infect Dis 203: 1282-1291, doi: jir012 [pii] 10.1093/infdis/jir012.

10. Friberg, H. et al. Memory CD8+ T cells from naturally acquired primary dengue virus infection are highly cross-reactive. Immunol Cell Biol 89: 122-129, doi: icb201061 [pii] 10.1038/icb.2010.61.

11. Bashyam, H.S., Green, S. \& Rothman, A.L. 2006. Dengue virus-reactive CD8+ T cells display quantitative and qualitative differences in their response to variant epitopes of heterologous viral serotypes. J Immunol 176: 2817-2824, doi: 176/5/2817 [pii].

12. Gunther, V.J. et al. 2011. A human challenge model for dengue infection reveals a possible protective role for sustained interferon gamma levels during the acute phase of illness. Vaccine 29: 3895-3904, doi: S0264410X(11)00406-3 [pii] 10.1016/j.vaccine. 2011.03.038.

13. Chaturvedi, U.C. 2009. Shift to Th2 cytokine response in dengue haemorrhagic fever. Indian J Med Res 129: 1-3.

14. Langenkamp, A., Messi, M., Lanzavecchia, A. \& Sallusto, F. 2000. Kinetics of dendritic cell activation: impact on priming of TH1, TH2 and nonpolarized T cells. Nat Immunol 1: 311-316, doi: 10.1038/79758.

15. Ying, S. et al. 2005. Thymic stromal lymphopoietin expression is increased in asthmatic airways and correlates with expression of Th2-attracting chemokines and disease severity. J Immunol 174: 8183-8190, doi: 174/ 12/8183 [pii].

16. Wang, Y.H. et al. 2006. Maintenance and polarization of human TH2 central memory T cells by thymic stromal lymphopoietinactivated dendritic cells. Immunity 24: 827838, doi: S1074-7613(06)00230-5 [pii] 10. 1016/j.immuni.2006.03.019.

17. Esnault, S., Rosenthal, L.A., Wang, D.S. \& Malter, J.S. 2008. Thymic stromal lymphopoietin (TSLP) as a bridge between infection and atopy. Int J Clin Exp Pathol 1: 325-330.

18. Miazgowicz, M.M., Headley, M.B., Larson, R.P. \& Ziegler, S.F. 2009. Thymic stromal lymphopoietin and the pathophysiology of atopic disease. Expert Rev Clin Immunol 5: 547-556, doi: 10.1586/eci.09.45.

19.Zhang, Z. et al. 2009. Thymic stromal lymphopoietin overproduced by keratinocytes in mouse skin aggravates experimental asthma. Proc Natl Acad Sci USA 106, 1536-1541, doi: 0812668106 [pii] 10.1073/ pnas. 0812668106.

20. Kashyap, M., Rochman, Y., Spolski, R., Samsel, L. \& Leonard, W.J. 2011. Thymic Stromal Lymphopoietin Is Produced by 
Dendritic Cells. J Immunol, doi: jimmunol. 1100355 [pii] 10.4049/jimmunol. 1100355.

21. Holgate, S.T. 2007. The epithelium takes centre stage in asthma and atopic dermatitis. Trends Immunol 28: 248-251, doi: S1471-4906 (07)00104-4 [pii] 10.1016/j.it.2007.04.007.

22. Allakhverdi, Z. et al. 2007. Thymic stromal lymphopoietin is released by human epithelial cells in response to microbes, trauma, or inflammation and potently activates mast cells. J Exp Med 204: 253-258, doi: jem. 20062211 [pii] 10.1084/jem.20062211.

23. Qiao, J., Li, A. \& Jin, X. TSLP from RSVstimulated rat airway epithelial cells activates myeloid dendritic cells. Immunol Cell Biol, doi: icb201085 [pii] 10.1038/ icb.2010.85.

24. Kato, A. \& Schleimer, R.P. 2007. Beyond inflammation: airway epithelial cells are at the interface of innate and adaptive immunity. Curr Opin Immunol 19: 711-720, doi:S0952-7915(07)00164-1 [pii] 10.1016/ j.coi.2007. 08.004.

25. Wang, J. \& Xing, F. 2008. Human TSLPeducated DCs. Cell Mol Immunol 5: 99-106, doi: $10.1038 / \mathrm{cmi} .2008 .12$.

26. Rimoldi, M. et al. 2005. Intestinal immune homeostasis is regulated by the crosstalk between epithelial cells and dendritic cells. Nat Immunol 6: 507-514, doi: ni1192 [pii] $10.1038 / \mathrm{ni} 1192$.

27. WHO. 2009. Dengue guidelines for diagnosis, treatment, prevention and control: new edition. World Health Organization Geneve.

28. Barniol, J. et al. Usefulness and applicability of the revised dengue case classification by disease: multi-centre study in 18 countries. BMC Infect Dis 11: 106, doi: 1471-2334-11106 [pii] 10.1186/1471-2334-11-106.

29. Fontenot, D. et al. 2009. TSLP production by epithelial cells exposed to immunodeficiency virus triggers $\mathrm{DC}$-mediated mucosal infection of CD4+ T cells. Proc Natl Acad Sci USA 106: 16776-16781, doi: 0907347106 [pii] 10.1073/ pnas.0907347106.

30. Lee, E.B. et al. 2009. Increased serum thymic stromal lymphopoietin in children with atopic dermatitis. Pediatr Allergy Immunol 21: e457-460, doi: PAI919 [pii] 10.1111/j.13993038.2009.00919.x.

31. Cooper, P. J. et al. 2011. Impact of early life exposures to geohelminth infections on the development of vaccine immunity, allergic sensitization, and allergic inflammatory diseases in children living in tropical Ecuador: the ECUAVIDA birth cohort study. BMC Infect Dis 11: 184, doi: 1471-2334-11-184 [pii] 10.1186/1471-2334-11-184.

32. Hunninghake, G. M. et al. 2010. TSLP polymorphisms are associated with asthma in a sex-specific fashion. Allergy 65: 15661575, doi:ALL2415 [pii] 10.1111/j.13989995.2010.02415.x.

33. Gunther, V. J. et al. 2011. A human challenge model for dengue infection reveals a possible protective role for sustained interferon gamma levels during the acute phase of illness. Vaccine 29: 3895-3904, doi:S0264$410 X(11) 00406-3$ [pii] 10.1016/j.vaccine. 2011.03.038.

34. Lu, N., Wang, Y.H., Arima, K., Hanabuchi, S. \& Liu, Y.J. 2009. TSLP and IL-7 use two different mechanisms to regulate human CD4+ T cell homeostasis. J Exp Med 206: 21112119, doi: jem.20090153 [pii] 10.1084/ jem.20090153.

35.Saenz, S.A., Taylor, B.C. \& Artis, D. 2008. Welcome to the neighborhood: epithelial cellderived cytokines license innate and adaptive immune responses at mucosal sites. Immunol Rev 226: 172-190, doi: IMR713 [pii] 10.1111/j.1600-065X.2008. 00713.x. 